\title{
Structural Design and Multi-objective Optimization of Planetary Frame for 6T40E Automatic Transmission
}

\author{
ZHAO Niu, HUANG Zhao-ming
}

\begin{abstract}
The 6T40E automatic transmission with three simple planetary rows and six shifting actuators can achieve six forward gears and one reverse gearing. Based on CATIA software and ANSYS software, this paper carries out three-dimensional modeling and finite element analysis of planetary frame, the key component of 6T40E. Taking the highest transfer efficiency and the smallest overall volume of planetary gear train as the ultimate optimization objective, a correct multi-objective optimization mathematical model of efficiency and volume is established. The multi-objective optimization of planetary gear train is carried out by using MATLAB software, which provides theoretical guidance for the optimal design of planetary gear transmission mechanism. It provides a theoretical basis for the researchers engaged in automotive and mechanical design to reasonably select transmission type, correctly design and apply, develop, test and develop new types.
\end{abstract}

Index Terms: 6T40E automatic transmission, finite element analysis, planetary gear train, multi-objective optimization

\section{INTRODUCTION}

In this paper, the automatic transmission of planetary gear mechanism is taken as the research object, and the structural characteristics of 6T40E multi-row planetary gear train are analyzed. At the same time, three-dimensional modeling and finite element analysis of the planetary frame, the key component of CATIA V5R17 and ANSYS are carried out. The ANSYS software is used to restrict the output of the planetary carrier, and the corresponding maximum load is applied to the inner walls of the holes of the planetary carrier, and the calculation is submitted. The stress and strain cloud pictures of the planetary carrier under the maximum load are obtained. Comparing the calculated results with the theoretical data, it is proved that the planetary frame model can satisfy the stress requirement of the planetary frame. Finally, the transmission efficiency of the planetary gear train is calculated, and the overall volume and transmission efficiency of the planetary gear train are optimized by using the optimization toolbox of MATLAB to achieve the optimal design of the structure of the planetary gear train ${ }^{[1]}$.

Zhao Niu, Wentian College, Hohai University, Ma'anshan, People's Repubilc of China

Huang Zhao-ming, Wentian College, Hohai University, Ma'anshan, People's Republic of China

\section{STRUCTURAL CHARACTERISTICS OF 6T40E PLANETARY GEAR TRAIN}

The 6T40E automatic transmission is used in the new Buick La Crosse, Buick English Lang, Chevrolet Cruz, Buick GL8 and Buick New Regal. It is an electrically controlled 6-speed automatic transmission. With only three simple planetary rows and six shifting actuators, it can form six forward gears and one reverse gear. The transmission can be shifted through the shift control keys on the steering wheel. It has the characteristics of compact structure, excellent performance and fuel saving, and can enable the vehicle to have low speed cruising ability ${ }^{[2]}$. The planetary gear mechanism and shift actuator are shown in Fig. 1. Its shift actuator consists of six clutches ${ }^{[3]}$.

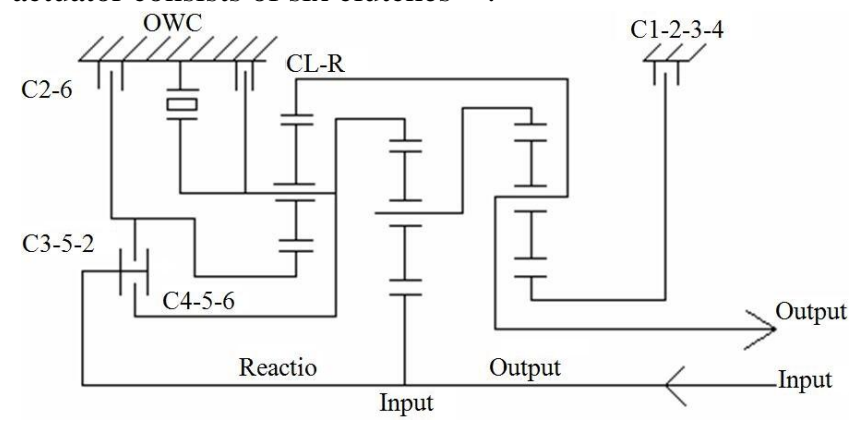

Fig.1 Planetary gear mechanism and shift actuators

\section{MODELING OF 6T40E KEY COMPONENTS}

This paper uses CATIA software to build 3D model of 6T40E key parts. The specific process is as follows:

Firstly, establish the reference plane of sketch drawing: select one plane in the three planes XY plane, YZ plane and $\mathrm{XZ}$ plane of entity design, and draw the sketch again. Click the Sketch Drawing button in the right toolbar of the form to enter sketch editing mode and draw the cross section of the planetary frame on the plane. Add various constraints: use button to constrain the dimensions of the sketch, and press to push out the sketch after complete constraints. The solid feature modeling is generated by using the convex button, and the contour line is selected as the working object, then the solid feature modeling is generated by using the convex button. Punch holes and chamfer on the planetary shelf, press and complete the final operation ${ }^{[4]}$. As shown in Figure 2.

\section{FINITE ELEMENT ANALYSIS OF PLANETARY FRAME}

In this paper, ANSYS software is used to carry out finite element analysis of $6 \mathrm{~T} 40 \mathrm{E}$ planetary carriage. The output 
terminal of the planetary carriage is constrained, and the corresponding maximum load is applied to the inner walls of the holes of the planetary carriage. The stress and strain nephograms of the planetary carriage under the maximum

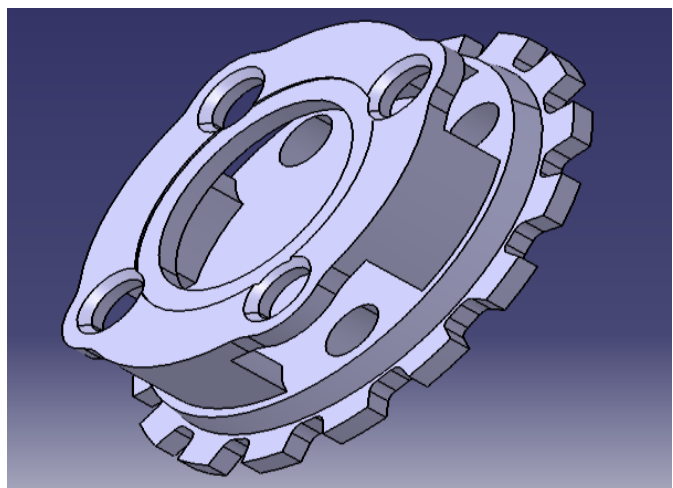

Fig.2 Planetary Frame of Automatic Transmission

load are obtained. Verify that the established planetary frame model meets its stress requirements ${ }^{[5]}$. First start ANSYS Workbench 14.0 and enter the main interface.

Double-click the main interface Toolbox Analysis Systems to Static Structural, Create analysis projects A. Import Create Geometry: Right-click on Geomeyry ${ }^{[6]}$. Select Browse in Import Geometry to import the geometry file. Adding Material Library: Double-click Engineering Data to set material parameters and select nodular cast iron for planetary frame material. Add model material attributes ${ }^{[7]}$ : Double-click Model, enter the Mechanical interface, select Propeller under Geometry in Outlines, model material attributes can be add. Meshing: Mesh is selected, the mesh type is tetrahedron, other mesh parameters are modified, and the mesh is divided ${ }^{[8]}$. The final effect is shown in Figure 3.

Load and constraints: Select Static Structural, then Displacement in Supports, and select the surface that needs to be constrained. Pressure is chosen as the load type with the sizes of $6.277 \mathrm{MPa}$ and $1.638 \mathrm{MPa}$ respectively. The output end, i.e. the output ring part ${ }^{[9]}$ is fixed. The results are shown in Fig.4and Fig.5.

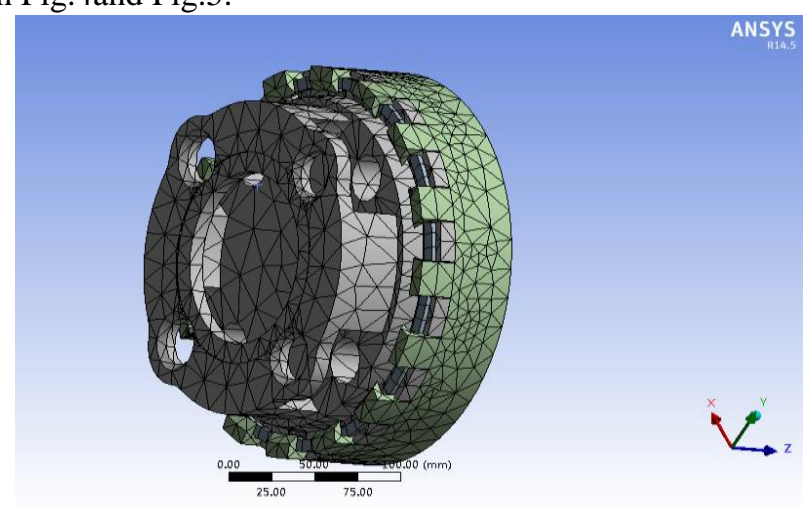

Fig.3 The effect of meshing

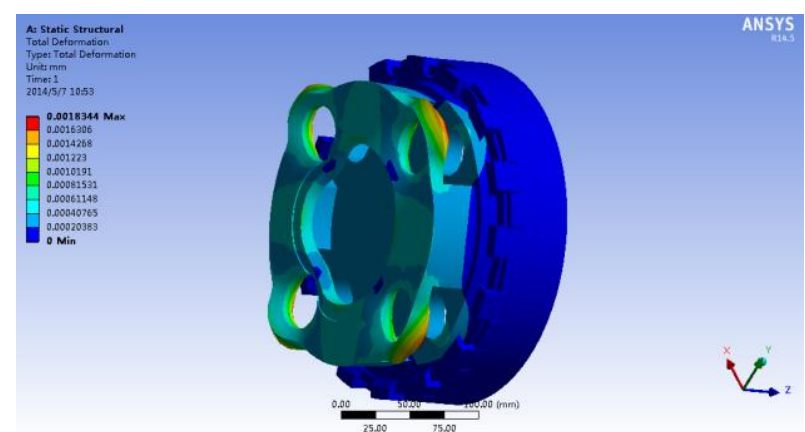

Fig. 4 Strain Diagram

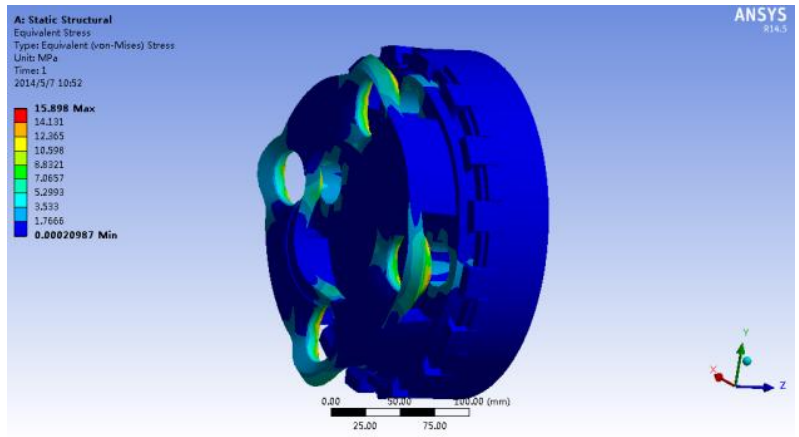

Fig.5 Stress Diagram

Result analysis: The finite element model of planetary frame is loaded and constrained by ANSYS, and the planetary frame is slightly deformed when it is subjected to force. The color of stress distribution in the diagram changes from blue to red, indicating that the stress changes from low to high, and the displacement changes from small to large on the displacement diagram.

The maximum load in the stress diagram is $15.898 \mathrm{MPa}$ and the maximum deformation in the strain diagram is 0.0018 $\mathrm{mm}$.

The extrusion stress is $19.721 \mathrm{MPa}$ calculated by traditional method and $15.898 \mathrm{MPa}$ calculated by finite element analysis.

The error analysis of the data calculated by the two methods is as follows, which shows that it meets the requirements.

\section{OPTIMUM DESIGN OF PLANETARY GEAR TRAIN BASED ON MATLAB}

The 6T40E automatic transmission designed in this paper, in order to achieve the ultimate optimization goal of the highest transmission efficiency and the smallest overall volume of the planetary gear train, the multi-objective optimization design of its planetary gear train is carried out by using the optimization toolbox of MATLAB, and the correct multi-objective optimization mathematical model of efficiency and volume is established. Combining with the case, the solving steps of the optimized parameters are given, which provides theoretical guidance for the optimal design of planetary gear transmission mechanism ${ }^{[10]}$.

\section{A. Object Function and Design Variables}

The main parameters affecting the volume of planetary gear are the volume of solar wheel $V_{\mathrm{a} 1}$ and $V_{\mathrm{a} 2}$, the volume of inner gear ring $V_{\mathrm{b} 1}, V_{\mathrm{b} 2}$ and the volume of planetary gear $V_{\mathrm{c} 1}$, 
World Journal of Research and Review (WJRR)

ISSN:2455-3956, Volume-8, Issue-6, June 2019 Pages 42-46

$V_{\mathrm{c} 2}$ and $V_{\mathrm{c} 3}$. The expression method of total volume $V_{\mathrm{s}}$ is obtained $^{[10-11]}$.

\section{一 、 Front row}

Sun gear 1 volume:

$V_{a 1} \approx \pi\left(\frac{d_{a 1}}{2}\right)^{2} \square B_{1}=\frac{\pi B_{1}}{4} d_{a 1}^{2}=\frac{\pi B_{1} m^{2}}{4} Z_{a 1}^{2}$

Planetary 1 volume:

$V_{c 1} \approx \pi\left(\frac{d_{c 1}}{2}\right)^{2} \square B_{1}=\frac{\pi B_{1}}{4} d_{c 1}^{2}=\frac{\pi B_{1} m^{2}}{4} Z_{c 1}^{2}$

Ring gear 1 volume:

$$
\begin{aligned}
& V_{b 1} \approx \pi\left(\frac{d_{b 1}+h_{f}+C}{2}\right)^{2} \square B_{1}-\pi\left(\frac{d_{b 1}}{2}\right)^{2} \square B_{1} \\
& h_{f}=\left(h_{a}^{*}+c^{*}\right) m
\end{aligned}
$$

Because we have the equation (4), $h_{a}^{*}$ is coefficient of crown height which equals $1 ; c^{*}$ is top clearance coefficient, which equals 0.25 , we could get :

$V_{b 1} \approx \frac{\pi}{4} B_{1} \square m_{1}^{2}\left(\left(Z_{b 1}+1.25+\frac{C}{m_{1}}\right)^{2}-Z_{b 1}^{2}\right)$

\section{二、Rear row}

Sun gear 2 volume :

$$
V_{a 2} \approx \pi\left(\frac{d_{a 2}}{2}\right)^{2} \square B_{2}=\frac{\pi B_{2}}{4} d_{a 2}^{2}=\frac{\pi B_{2} m^{2}}{4} Z_{a 2}^{2}
$$

(6)

Planetary gear 2 volume :

$$
V_{c 2} \approx \pi\left(\frac{d_{c 2}}{2}\right)^{2} \square B_{2}=\frac{\pi B_{2}}{4} d_{c 2}^{2}=\frac{\pi B_{2} m^{2}}{4} Z_{c 2}^{2}
$$

Gear ring 2 volume :

$$
\begin{aligned}
& V_{b 2} \approx \frac{\pi}{4} B_{2} \square m_{2}^{2}\left(\left(Z_{b 2}+1.25+\frac{C}{m_{2}}\right)^{2}-Z_{b 2}^{2}\right) \\
& V_{s}=V_{a 1}+V_{a 2}+V_{b 1}+V_{b 2}+V_{c 1}+V_{c 2}+V_{c 3} \\
& V_{s} \approx \sum_{i=1}^{2} \frac{\pi}{4} B_{i} m_{i}^{2}\left(Z_{a i}^{2}+\left(Z_{b i}+1.25+\frac{C}{m_{i}}\right)^{2}-Z_{b i}^{2}\right) \\
& +\sum_{i=1}^{3} \frac{\pi}{4} B_{i} m_{i}^{2}\left(n_{i} Z_{c i}^{2}\right)
\end{aligned}
$$

In the above formula, $\mathrm{B}, \mathrm{m}, \mathrm{N}$ and $\mathrm{C}$ represent the outer ring thickness of tooth width, magic and number of gears respectively, $Z_{\mathrm{a}}, Z_{\mathrm{b}}$ and $Z_{\mathrm{c}}$ represent the number of teeth of sun gear, inner ring gear and planetary gear respectively ${ }^{[12]}$.

\section{B. Efficiency}

Because the designed double-row planetary gear train is a two-stage planetary gear train, the total transmission efficiency $\eta_{\mathrm{p}}$ of the planetary gear train is determined by the product of the transmission efficiency $\eta_{1}$ and $\eta_{2}$ of the two-stage planetary gear train. Therefore, the formula for calculating transmission efficiency is as follows:

$\eta_{p}=\left(1-\frac{p_{1}}{1+p_{1}} \varphi^{H 1}\right)\left(1-\frac{p_{2}}{1+p_{2}} \varphi^{H 2}\right)$

In this equation

$$
\begin{aligned}
& P_{1}=\frac{z_{b 1}}{z_{a 1}} \\
& P_{2}=\frac{z_{b 2}}{z_{a 2}}
\end{aligned}
$$

Which $P_{1} 、 P_{2}$ are characteristic parameters of two-stage planetary gear mechanism (internal transmission ratio), $\varphi^{\mathrm{H} 1} 、 \varphi^{\mathrm{H} 2}$ are the loss coefficient of two-stage planetary gear.

$\varphi^{H 1}=\varphi_{2 a}^{H}+\varphi_{2 b}^{H}+\varphi_{n}^{H}$

The external and internal mesh loss coefficients are:

$\varphi_{2 a}^{H}=\frac{\pi}{2} \varepsilon f_{k}\left(\frac{1}{z_{a}}+\frac{1}{z_{c}}\right)$

$\varphi_{2 a}^{H}=\frac{\pi}{2} \varepsilon f_{k}\left(\frac{1}{z_{c}}-\frac{1}{z_{b}}\right)$

Where $\varepsilon$ is coincidence degree, $f_{\mathrm{k}}$ is the meshing friction factor, $\varphi_{\mathrm{n}}{ }^{\mathrm{H}}$ is bearing loss coefficient ${ }^{[13]}$.

\section{Design variable}

The first objective function can be obtained by introducing the optimal design variables into the volume formula.

$$
\begin{aligned}
& f_{1}(x)=\frac{\pi}{4} x_{1} x_{3}^{2}\left[x_{5}^{2}+n_{1} x_{7}^{2}+\left(x_{6}+1.25+C / x_{3}\right)^{2}-x_{6}^{2}\right] \\
& +\frac{\pi}{4} x_{2} x_{4}^{2}\left[x_{8}^{2}+n_{2} x_{10}^{2}+n_{2} x_{11}^{2}+\left(x_{9}+1.25+C / x_{4}\right)^{2}-x_{9}^{2}\right]
\end{aligned}
$$

The second objective function is obtained by substituting the efficiency formula.

$$
\begin{aligned}
& f_{2}(x)=\left\{1-x_{6} /\left(x_{5}+x_{6}\right)\left[\frac{\pi}{2} \varepsilon f_{k}\left(1 / x_{5}+2 / x_{7}+1 / x_{6}\right)+\varphi_{n}{ }^{H}\right]\right\} \\
& \times\left\{1-x_{9} /\left(x_{8}+x_{9}\right)\left[\frac{\pi}{2} \varepsilon f_{k}\left(1 / x_{8}+2 / x_{10}+2 / x_{11}-1 / x_{9}\right)+\varphi_{n}{ }^{H}\right]\right\}
\end{aligned}
$$

\section{Constraint Condition}

\section{Drive Ratio Conditions}

$$
\begin{aligned}
& \left|\frac{\left(Z_{a 1}+Z_{b 1}\right)\left(Z_{a 2}+Z_{b 2}\right)}{Z_{a 1} Z_{a 2} i_{p}}-1\right|<4 \% \\
& g_{1}(x)=\left|\frac{\left(x_{5}+x_{6}\right)\left(x_{8}+x_{9}\right)}{x_{5} x_{8} i_{p}}-1\right|-4 \%<0
\end{aligned}
$$

Where $i_{\mathrm{p}}$ is the total transmission ratio of two-stage planetary gears. 


\section{Adjacency condition}

$$
\begin{aligned}
& d_{a 1 c 1}<2 a_{a 1 c 1} \sin \left(\pi / n_{1}\right) \\
& 2 h_{a}^{*}+Z_{c 1}<\left(Z_{a 1}+Z_{c 1}\right) \sin \frac{\pi}{n_{1}} \\
& g_{2}(x)=2 h_{a}^{*}+x_{7}-\left(x_{5}+x_{7}\right) \sin \frac{\pi}{n_{1}}<0
\end{aligned}
$$

$$
\begin{aligned}
& d_{a 2 c 3}<2\left(a_{a 2 c 2}+a_{c 2 c 3}\right) \sin \left(\pi / n_{2}\right) \\
& 2 h_{a}^{*}+Z_{c 3}<\left(Z_{a 2}+2 Z_{c 2}+Z_{c 3}\right) \sin \frac{\pi}{n_{2}} \\
& g_{3}(x)=2 h_{a}^{*}+x_{11}-\left(x_{8}+2 x_{10}+x_{11}\right) \sin \frac{\pi}{n_{2}}<0
\end{aligned}
$$

Where $d_{\mathrm{ac}}$ is the top circle of the planetary gear and the solar gear, $a_{\mathrm{ac}}$ is the center distance of the meshing pair between the solar gear and the planetary gear, and $h_{\mathrm{a}}$ is the top height coefficient.

\section{Contact strength condition}

$$
\begin{aligned}
& Z_{H} Z_{E} Z_{\delta} \sqrt{\frac{F_{t 1}}{d_{1} B_{1} n_{1}} \frac{u_{1}-1}{u_{1}}}-\delta_{H O} \leq 0 \\
& g_{4}(x)=Z_{H} Z_{E} Z_{\delta} \sqrt{\frac{2 T_{1}}{x_{3}^{2} x_{5}^{2} x_{1} n_{1}} \frac{x_{6}-x_{5}}{x_{6}}}-\delta_{H O} \leq 0 \\
& Z_{H} Z_{E} Z_{\delta} \sqrt{\frac{F_{t 2}}{d_{2} B_{2} n_{2}} \frac{u_{2}-1}{u_{2}}}-\delta_{H O} \leq 0 \\
& g_{5}(x)=Z_{H} Z_{E} Z_{\delta} \sqrt{\frac{2 T_{2}}{x_{4}^{2} x_{8}^{2} x_{2} n_{2}} \frac{x_{9}-x_{8}}{x_{9}}}-\delta_{H O} \leq 0
\end{aligned}
$$

Where $Z_{\mathrm{H}}$ is the node area coefficient ; $Z_{\mathrm{E}}$ is elastic influence coefficient $; Z_{\delta}$ contact ratio factor $; T_{1} 、 T_{2}$ are torque transferred by two-stage pinion gears ; $\delta_{\mathrm{HO}}$ is allowable contact stress ; $F_{\mathrm{t} 1} 、 F_{\mathrm{t} 2}$ are tangential forces acting on pinion gears at all levels; $d_{1} 、 d_{2}$ are dividing circle diameter of pinion at all levels; $u_{2}$ are high speed transmission ratio ; $u_{2}$ is low speed stage transmission ratio ${ }^{[14]}$ 。

Bending Strength Conditions

$$
\begin{aligned}
& \frac{F_{t 1} Y_{F a} Y_{S a} Y_{\varepsilon}}{B_{1} m_{1} n_{1}}-\delta_{F o} \leq 0 \\
& g_{6}(x)=\frac{2 T_{1} Y_{F a} Y_{S a} Y_{\varepsilon}}{x_{3}^{2} x_{5} x_{1} n_{1}}-\delta_{F o} \leq 0 \\
& \frac{F_{t 2} Y_{F a} Y_{S a} Y_{\varepsilon}}{B_{2} m_{2} n_{2}}-\delta_{F o} \leq 0 \\
& g_{7}(x)=\frac{2 T_{2} Y_{F a} Y_{S a} Y \varepsilon}{x_{4}^{2} x_{8} x_{2} n_{2}}-\delta_{F 0} \leq 0
\end{aligned}
$$

Where $Y_{\varepsilon}$ is contact ratio factor; $Y_{\mathrm{Sa}}$ is stress correction coefficient; $Y_{\mathrm{Fa}}$ is tooth profile coefficient; $\delta_{\mathrm{F} 0}$ is allowable bending stress.

\section{External Diameter Size Conditions}

The design of two-stage planetary gears requires that the diameters of the two inner rings be similar to each other to minimize the radial dimensions. A parameter is introduced to represent the ratio of ring diameter between high-speed stage and low-speed stage, which is

$$
C_{0}=\frac{d_{1}}{d_{2}}
$$

Commonly $0.8 \leq C_{0} \leq 1.2$, we have

$0.8 \leq C_{0} \leq 1.2$

$g_{8}(x)=0.8-\frac{x_{3} x_{6}}{x_{4} x_{9}} \leq 0$

$C_{0}=\frac{d_{1}}{d_{2}}=\frac{m_{1} Z_{b 1}}{m_{2} Z_{b 2}}$

z $g_{9}(x)=\frac{x_{3} x_{6}}{x_{4} x_{9}}-1.2 \leq 0$

\section{Concentric Condition}

$Z_{a 1}+2 Z_{c 1}=Z_{b 1}$

$g_{10}(x)=x_{5}+2 x_{7}-x_{6}=0$

Installation Conditions

$\frac{Z_{a 1}+Z_{b 1}}{n_{1}}=C_{1}$

$h_{11}(x)=\frac{x_{5}+x_{6}}{n_{1}}-C_{1}=0$

$\frac{Z_{a 2}+Z_{b 2}}{n_{2}}=C_{2}$

$h_{12}(x)=\frac{x_{8}+x_{9}}{n_{2}}-C_{2}=0$

Where $C_{1}, C_{2}$ are positive integer.

\section{AN EXAMPLE OF OPTIMUM DESIGN}

An example of optimization design is used to solve the calculation process of multi-objective programming by goal-attainment method. It can be realized by calling "fgoalattain" function in optimization toolbox of MATLAB software system. This function is mainly applied to the optimization of multi-objective problems, and the following optimization results are obtained ${ }^{[15]}$. 


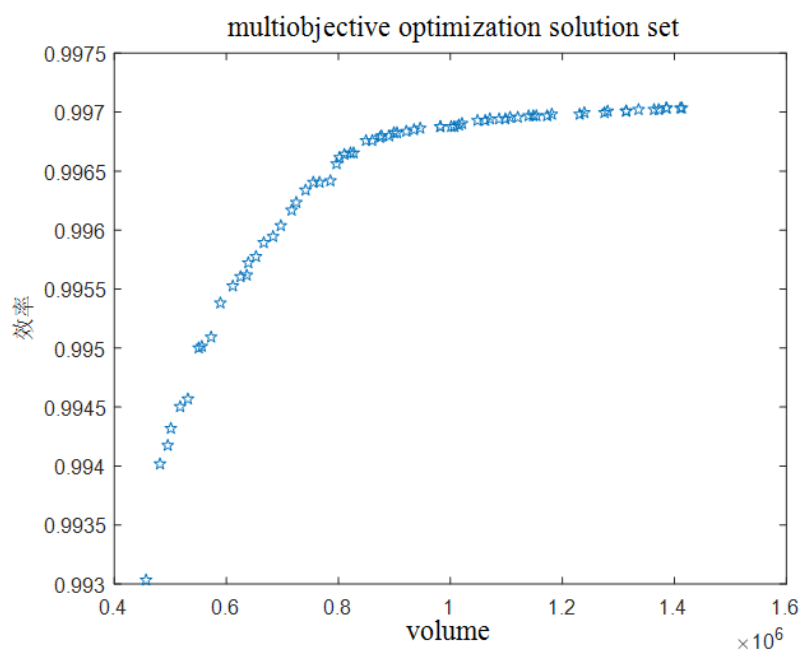

Fig. 6 Optimized Volume Efficiency Diagram

It can be seen that the overall volume of planetary gear train is reduced by $29.01 \%$ by optimizing the design parameters of the toolbox of MATLAB. After optimization, the efficiency is increased by $0.601 \%$ which achieves the design objectives. The optimized volume efficiency diagram is shown in Fig.6.

\section{CONCLUSION}

1. In this paper, through CATIA and ANSYS software, the key components of 6T40E automatic transmission are modeled in three dimensions, and the finite element analysis is carried out. The maximum load and deformation are obtained, which verifies that the design requirements are met.

2. By using the optimization toolbox of MATLAB, the multi-objective optimization design of its planetary gear train is carried out. Taking the highest transmission efficiency and the smallest overall volume of the planetary gear train as the ultimate optimization objective, the correct multi-objective optimization mathematical model of efficiency and volume is established, which provides theoretical guidance for the optimization design of planetary gear transmission mechanism.

\section{ACKNOWLEDGMENT}

This work was supported by Anhui Provincial Academic (Professional) top-notch talents academic funding project, The first author acts as responsible person (gxbjZD63).

\section{REFERENCES}

[1] Peng Z, Yingfu G, Houcai L . Multi-objective Optimization Design of Planetary Gear Reducer[J]. Mechanical Engineer, 2014.

[2] Lin T . Dynamic Performance Optimization of Coupled System for Four-stage Planetary Gear Reducer[J]. Journal of Mechanical Engineering, 2018, 54(11):161.

[3] Zhang J , Qin X, Xie C , et al. Optimization design on dynamic load sharing performance for an in-wheel motor speed reducer based on genetic algorithm[J]. Mechanism and Machine Theory, 2018, 122:132-147.

[4] Zhang, Jiang, et al. "Optimization design on dynamic load sharing performance for an in-wheel motor speed reducer based on genetic algorithm." Mechanism and Machine Theory122(2018):132-147.

[5] Kang H , Jiaqi M A , Gongchuan X , et al. Interval multi-objective optimization for the specific power of a helicopter's main reducer planetary gear trains[J]. Journal of Aerospace Power, 2017.

[6] A N. Zongquan, Huang Z, Wang L. Simulation and Optimization of Drawing Process for Radiator Cross Beam[J]. Journal of Jiujiang University, 2017.

[7] Kolar J W , Friedli T , Krismer F, et al. Conceptualization and multi-objective optimization of the electric system of an Airborne Wind Turbine[J]. IEEE Journal of Emerging \& Selected Topics in Power Electronics, 2013, 1(2):73-103.

[8] Bafandeh A, Bin-Karim S, Baheri A, et al. A comparative assessment of hierarchical control structures for spatiotemporally-varying systems, with application to airborne wind energy[J]. Control Engineering Practice, 2018, 74:71-83.

[9] Rancourt D , Bolduc-Teasdale F , Bouchard E D, et al. Design space exploration of gyrocopter-type airborne wind turbines[J]. Wind Energy, 2016, 19(5):895-909.

[10] Rancourt D , Bolduc-Teasdale F , Bouchard E D , et al. Design space exploration of gyrocopter-type airborne wind turbines[J]. Wind Energy, 2016, 19(5):895-909.

[11] Subotic I, Gammeter C , Tuysuz A, et al. Weight optimization of an axial-flux PM machine for airborne wind turbines[C]// 2016 IEEE International Conference on Power Electronics, Drives and Energy Systems (PEDES). IEEE, 2016.

[12] Zhang Z , Nilssen R , Muyeen S M , et al. Design optimization of ironless multi-stage axial-flux permanent magnet generators for offshore wind turbines $[\mathrm{J}]$. Engineering Optimization, 2016, 49(5):1-13.

[13] Zhou R , Li G , Ju L . Optimization design of pump motor based on genetic algorithm and neural network[C]// Industrial Electronics \& Applications. IEEE, 2016.

[14] Pei X , Zhou Y, Sheng Z . Torque ripple suppression of a new in-wheel motor based on quantum genetic algorithm[C]// International

[15] Ge L Z, Chen J , Li R, et al. Optimization Design of Drive System for Industrial Robots Based on Dynamic Performance[J]. Industrial Robot: An International Journal, 2017:00-00.

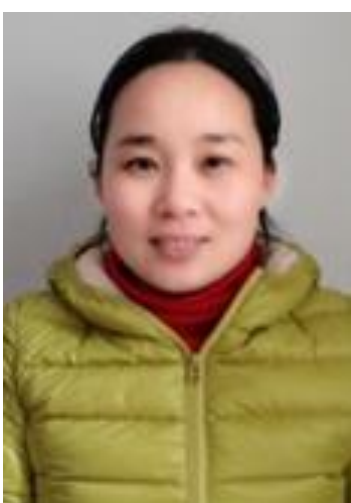

authorized.

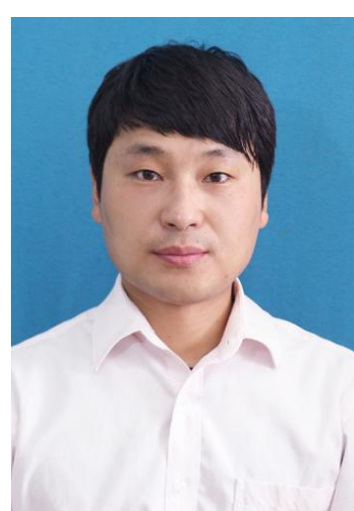

\section{Lecturer. ZHAO Niu}

\section{University Teachers CHINA}

ZHAO Niu: female, lecture, master, graduated from Nanjing University of Technology, major in transportation engineering, she is research direction is the theory and method of Modern vehicle design, she is in charge of automobile marketing and Automotive Electrical Appliances in Wanjiang University of Technology, she guided students to participate in the provincial level two prize 1 hosted 2 provincial-level topics, 2 edited textbooks, scientific research as the first author or correspondent author, 7 papers are published and more than 6 patents are

10 key scientific research projects at the provincial level, published more than 30 papers of three types or above by the first author or communication author, and authorized more than 20 patents. Social positions related to scientific research: Director of Anhui Engineering Graphics Society, Member of Ma'anshan Science and Technology Association. 